\title{
High-energy particles from SN-explosions near the Galactic center
}

\author{
Ernst A. Dorfi and Daniel Steiner \\ Institut für Astrophysik, Universität Wien \\ Türkenschanzstr. 17, A-1180 Wien, Austria \\ email: ernst.dorfi@univie.ac.at \\ email: daniel.steiner@univie.ac.at
}

\begin{abstract}
Several supernovae exploding in a compact cluster of massive stars generate a galactic outflow with embedded shock waves. Based on numerical simulations for an expanding superbubble above the Galactic center we find that these individual waves generated by the repeated SN-explosions, interact with each other and finally coalesce into a single strong shock at a distance of $5 \mathrm{kpc}$ above the Galactic plane at about $5 \cdot 10^{6}$ years after outbreak. The resulting shock with a Mach number $M \simeq 10$ propagates up to $100 \mathrm{kpc}$ in less than $10^{8}$ years. The time-dependent mass an energy loss out of the superbubble affects the further evolution of the outflow. In such long lasting shock waves energetic particles can be accelerated above the knee of $10^{15} \mathrm{eV}$ already near the galactic plane by a first-order Fermi-mechanism. The additional pressure gradients from such cosmic rays lead to further accelerations of the galactic outflow since these ultra-relativistic particles suffer less from adiabatic losses than the thermal gas.
\end{abstract}

Keywords. Supernova remnants, cosmic rays, particle acceleration, shock waves

\section{Introduction}

High energy particles are accelerated in shock waves by the so-called first-order Fermimechanism (e.g. review by Drury 1983), where charged particles are scattered back and forth at magnetic irregularities between the up- and downstream regions. Clearly, the gyro-radii of these particles have to be smaller than the typical size of the physical system. Shock waves within the ISM produced by SN-explosions are believed to be responsible for cosmic rays up $10^{14} \mathrm{eV}$, the so-called 'knee' of the particle distribution function observed at Earth. We propose that SN-explosions from OB-association in the vicinity of the Galactic center (GC) generate a large scale galactic outflow. The combined effect of thermal pressure, cosmic ray and wave pressure is able to push material out of the Galactic potential into the intergalactic space (e.g., Breitschwerdt et al. 1991). Shock waves within such structures re-accelerate the cosmic rays in regions less than $10 \mathrm{kpc}$ from the Galactic plane. Shock waves from different SNe merge to a single wave propagating outwards which can than boost the particles to higher energies, even up to $10^{19} \mathrm{eV}$. The high level of activity in the GC generates an ideal site for particle acceleration accompanied by frequent collisions with the thermal gas producing $\pi_{0}$ 's which decay into $\gamma$-rays.

\section{Physical and numerical models}

Starting with a Kompaneets (1960) solution we calculate the evolution of a superbubble (SB) within an exponential density stratification until outbreak. We adopt a scale-height of $h=50 p c$ and a particle density at the galactic plane of $n_{0}=10 \mathrm{~cm}^{-3}$. Typically nine SN-explosions are necessary to open the bubble into the intergalactic space which determines the volume as well as the inner boundary conditions at the bottom of the 

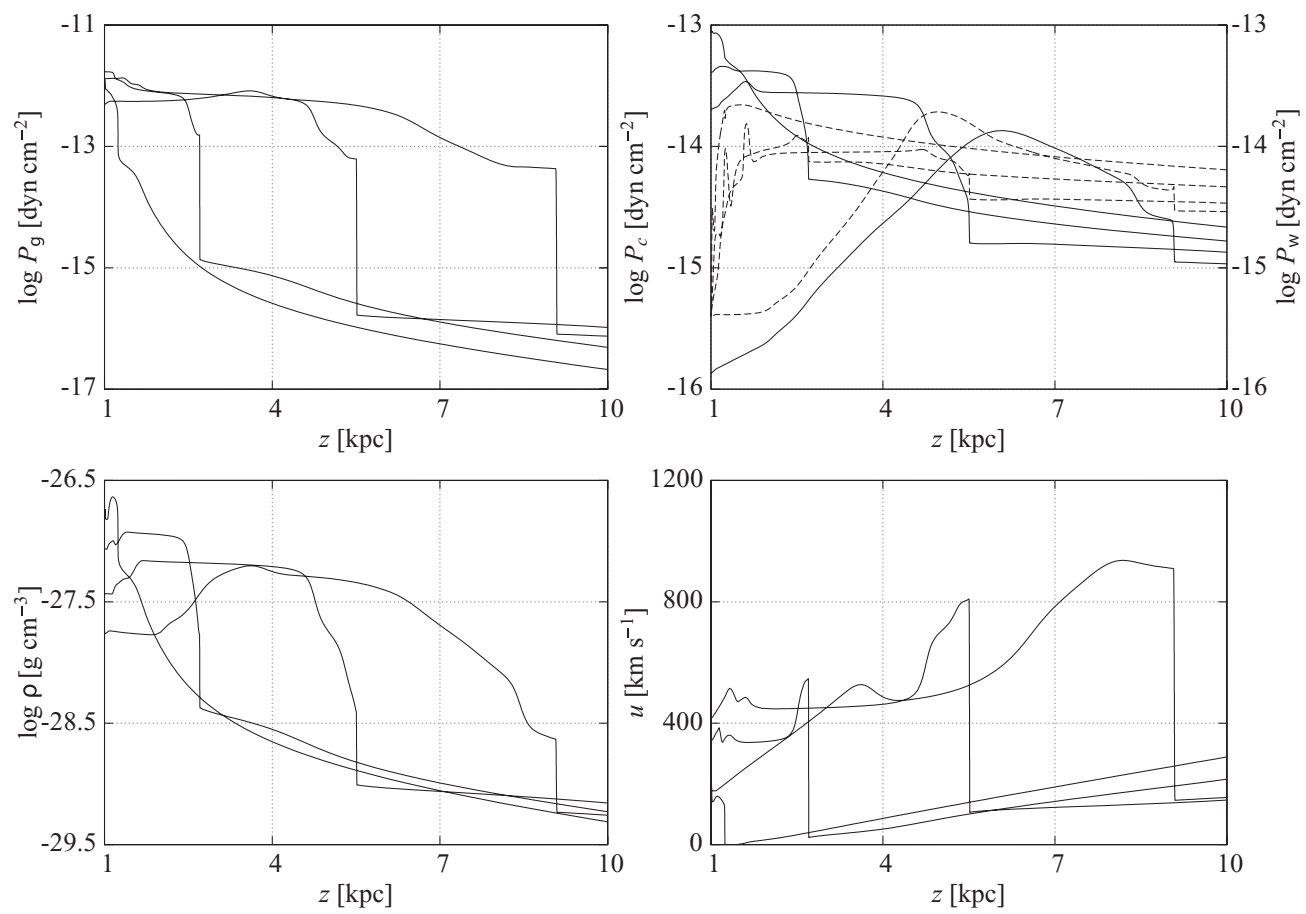

Figure 1. Time-dependent radial flow structures dominated by the forward shock wave are displayed between 1 and $10 \mathrm{kpc}$ at 4 ages, $3 \cdot 10^{6}, 6 \cdot 10^{6}, 9 \cdot 10^{6}$ and $1.2 \cdot 10^{7}$ years, respectively.

flux tube. The area of the cross-section $A_{0}$ of the superbubble is $A_{0}=\pi R^{2}=38800 p c^{2}$, corresponding to $R=110 \mathrm{pc}$. The OB-association is characterized by $25 \mathrm{SNe}$ within $1.2 \cdot 10^{7}$ years, each exploding with $10^{51} \mathrm{erg}$. Using an implicit numerical code (Dorfi \& Breitschwerdt 2013) we follow the flow structure in a prescribed flux-tube geometry up to $300 \mathrm{kpc}$, where the outer boundary is located. The pressure gradients of thermal gas, cosmic rays and waves accelerate a galactic outflow and thereby also reduce the energies within the superbubble. Each subsequent SN-explosions adds energy and momentum to the flow through propagating shock waves. The time-dependent evolution of such a galactic wind is plotted in Figure 1.

\section{Conclusions}

The coalescence of shock waves into a single wave occurs typically at a distance of $5 \mathrm{kpc}$, outflow velocities are of the order of $1000 \mathrm{~km} \mathrm{~s}^{-1}$. For a single OB-association the outflow basically ceases after the last SN-explosion at about $20 \cdot 10^{7}$ years, because the reverse shock has propagated back to the galactic superbubble. High energetic cosmic rays are re-accelerated at the shock waves and provide an additional pressure source for driving large scale galactic outflows.

\section{References}

Breitschwerdt D., McKenzie J. F., Völk H. J. 1991, A\&\&A 245, 79

Dorfi, E. A., Breitschwerdt D. 2013, A\& A 540, A77

Drury L. O.'C. 1983, Rep. Prog. Phys. 46, 973

Kompaneets A. S. 1960, Soviet Phys. Doklady 5, 46 